\title{
Retos a la Educación Peruana en el Siglo XXI
}

\section{Challenges to Peruvian Education in XXI ${ }^{\text {st }}$ Century}

\author{
Arturo Mario Rojas Huerta \\ Universidad César Vallejo
}

\begin{abstract}
Las nuevas tendencias educativas y la necesidad del uso de las nuevas tecnologías educativas en el currículo y en las prácticas pedagógicas, hace que la escuela peruana necesite adecuarse a estos cambios para que los futuros ciudadanos den respuestas efectivas a las características y demandas de la sociedad actual, y dejar atrás la educación del siglo XIX que todavía impera en las escuelas peruanas.

Por lo que el presente artículo analiza las características más relevantes de la sociedad del siglo XXI, la relación de la escuela peruana con las características de esta sociedad, la formación y el rol que debe tener el docente y al final se caracteriza el tipo de escuela que se requiere para que se responda a lo que demanda la sociedad actual, y en la que las facultades de educación de las universidades desempeñarían un rol preponderante.
\end{abstract}

Descriptores: Tecnología educacional, Formación de docentes, Tendencias educativas, Sistema educacional, Modelos educativos.

The new educational trends and the needs of the use of new educational technologies in the curriculum and teaching practices, the Peruvian schools must adapt to these changes so that the future citizens give effective responses to the characteristics and demands of current society and leave behind the nineteenth century that still prevails in Peruvian schools.

So this paper analyzes the most relevant characteristics of the knowledge society, the relation between the school and the characteristics of this society, the teacher education and the role of teachers and at the end it mentions what school is required to respond to this demand that today's society, and in which the education faculties of universities have a major role.

Keywords: Educational technologies, Teacher education, Educational trends, Educational system, Educational models.

*Contacto: samsa002@hotmail.com

ISSN: 1696-4713

rinace.net/reice/

revistas.uam.es/reice
Recibido: $\quad 14$ de noviembre 2014

$1{ }^{\text {a }}$ Evaluación: 13 de marzo de 2015

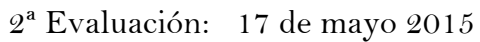

Aceptado: 15 de junio 2015 


\section{Introducción}

En la sociedad actual, se necesita que la escuela forme un tipo de persona que se adapte con rapidez a las nuevas circunstancias que nos impone la sociedad del siglo XXI, que sea capaz de iniciativa propia, que tenga la capacidad de aprender a aprender y que estén sensitivamente sintonizados con las personas que los rodean.

Hoy el conocimiento ya no es aplicable solo al ser sino más bien al hacer, pues este más que un recurso se convirtió en una utilidad. Sin embargo, las escuelas peruanas todavía están inmersas en el viejo orden industrial de la enseñanza en masa y un solo aprendizaje para todos, que no consigue atender con éxito a un alumnado heterogéneo y complejo, alumnado que ha cambiado mucho más que las instituciones diseñadas hace tiempo para acogerlo y formarlo para su posterior contribución a la sociedad. Entonces es necesario tener en cuenta lo expresado por Casanova, (2012): "la sociedad avanza y la educación no puede ni debe quedarse atrás si pretende preparar para la vida a las jóvenes generaciones" (p. 9).

En este artículo para apreciar la relación de la escuela peruana con las características y demandas de la sociedad actual, se analizó sus características más relevantes, la formación del estudiante y el rol del docente y además se pretendió caracterizar el tipo de escuela que se requiere para que responda a lo que se demanda en la actualidad y que por lo tanto haya una educación pertinente y de calidad.

\section{Características de la Sociedad del Siglo XXI}

La sociedad actual, que es la sociedad del conocimiento, está caracterizada por un crecimiento exponencial del conocimiento humano, por los continuos avances científicos y por una tendencia cada vez más a la globalización económica y cultural (gran mercado mundial, pensamiento único neoliberal, apogeo tecnológico, digitalización de toda la información). Cuenta con una difusión masiva de la informática, la telemática y los medios audiovisuales de comunicación en todos los estratos sociales y económicos, a través de los cuales nos proporciona: nuevos canales de comunicación (redes) e inmensas fuentes de información; potentes instrumentos para el proceso de la información; el dinero electrónico, nuevos valores y pautas de comportamiento social; nuevas simbologías y formas de organizar la información, configurando así nuestras visiones del mundo en el que vivimos e influyendo por lo tanto en nuestros comportamientos.

La sociedad del conocimiento enfatiza en la importancia de la elaboración de conocimiento funcional a partir de la información disponible, pues como lo plantea Gibbons y sus colaboradores (1998) "la producción del conocimiento tiene como finalidad el ser útil a alguien, sea en la industria, el gobierno, o la sociedad en general cada vez es menos la curiosidad la que le sirve de impulso a la investigación” (pp. 2-3).

Drucker (1994, pp. 6-7), por su parte, expresa que el conocimiento "es el único recurso significativo y dominante". Los tradicionales factores de la producción como la tierra (es decir, los recursos naturales), el trabajo y el capital- no han desaparecido, pero han pasado a ser secundarios. Se pueden obtener fácilmente, siempre que se tenga conocimiento. $\mathrm{Y}$ el conocimiento en este nuevo sentido es conocimiento como instrumento, como el medio de obtener resultados sociales y económicos. Además 
agrega que para el año 2000 no habrá ningún país desarrollado en que los trabajadores tradicionales que hacen y mueven bienes constituyan más de una sexta o una octava parte de la fuerza laboral.

Por su parte Tünnermann (2012) expresa:

Existe una tendencia irreversible a la desmaterialización del proceso productivo, hasta el punto que se afirma que el siglo XXI es el siglo del derrumbe de la materia, pues hay cada vez menos uso de materias primas y una mayor incorporación de los llamados intangibles, es decir conocimiento e información. Se habla así de una economía del saber. (p. 138)

La riqueza de las naciones ya no está en las mercancías tangibles o materias primas sino en la creación de nuevos conocimientos, y esto lo demuestra que más del 50\% del PIB de las mayores economías de la OECD (Organización para Cooperación y Desarrollo Económico) se halla ahora basado en conocimientos sobre todo en lo referente a los campos de la informática, biotecnología, ingeniería genética, microelectrónica, robótica, la industria espacial, entre otros.

A pesar de estas características de la sociedad actual, la escuela peruana es una institución anquilosada en el viejo orden industrial de la enseñanza en masa y el aprendizaje estandarizado, pues es ajena a los cambios que se dan en el siglo XXI pues se sigue utilizando el mismo enfoque pedagógico del siglo XIX que responde a una sociedad industrial. Por lo que la escuela peruana necesita asumir los nuevos paradigmas y modelos educativos y del uso de las Nuevas Tecnologías de Información y Comunicación (NTICs) en el currículo y en las prácticas pedagógicas en el aula de clases, para que así los futuros ciudadanos den respuestas efectivas a las características y demandas de la sociedad actual que es la del conocimiento, y dejar atrás la educación del siglo XIX que todavía impera en las escuelas peruanas y sobre todo en las autollamadas escuelas preuniversitarias, que dan más énfasis a la homogenización (que todos seamos más o menos parecidos en cuanto a formación básica), el enciclopedismo (que existe una verdad, que esa verdad la tienen algunos y que el resto debe ilustrarse), un sistema diseñado básicamente con la premisa one-size-fits-all («un modelo para todos») o lo que llamaría Freire (1970, pp. 51-52) una educación "bancaria” en la que los alumnos reciben de manera dócil contenidos de una programación establecida y totalmente cerrada, y mientras más la memorizan y repiten y más se dejan "llenar" tanto mejores alumnos serán, y el mejor docente será quien sea una "enciclopedia andante" y que pueda llenar lo más posible los depósitos de los alumnos.

Según lo plantea UNESCO-OREALC (2013) "los sistemas escolares se ven enfrentados a la necesidad de una transformación mayor e ineludible de evolucionar desde una educación que servía a una sociedad industrial, a otra que prepare para desenvolverse en la sociedad del conocimiento"(p. 10).

La escuela peruana ante todo lo anterior no estaría dando respuestas a las nuevas demandas y características de la sociedad actual y de los estudiantes, por lo que se consideraría que brinda una educación no pertinente y por lo tanto de poca calidad, siendo todo lo contrario de lo que se pregonan desde las escuelas preuniversitarias y desde otros organismos gubernamentales y no gubernamentales, por lo que hay una gran incoherencia entre el discurso educativo, el currículo implementado, y la metodología de los docentes en el aula de clases.

El acceso a una educación de calidad, en tanto derecho fundamental de todas las personas, como ya se mencionó, se enfrenta a un contexto de cambio paradigmático en el 
siglo XXI. El desarrollo que han alcanzado las NTICs demanda al sistema educacional una actualización de prácticas y contenidos que sean acordes a la nueva sociedad del siglo XXI, y que estos vayan delineados en el currículo nacional.

La escuela debe reaccionar y darse cuenta que no sólo vivimos un cambio de época sino una época de cambios, pero es muy claro que los cambios que se produzcan en las escuelas deben venir desde las universidades, precisamente de las facultades de educación, pues la universidad y sus distintas funciones deben estar orientadas en buscar el desarrollo humano sostenible y lograr de ese modo sociedades más prósperas y más justas, pero mayormente las universidades son, según Tünnermann (2011) torres de marfil que están de espaldas y se desentienden en gran medida de las problemáticas que suceden en la sociedad y en la mayoría de los casos no contribuyen al desarrollo del conjunto del sistema educativo.

\section{Las Escuelas Peruanas en el Siglo XXI}

Las escuelas han ido cambiando y modificándose según las necesidades y retos que le plantea la propia sociedad, sin embargo en el Perú se aprecia una parálisis paradigmática, tanto en las instituciones educativas como en la sociedad, de aceptar el cambio, pues todavía se enseña como en el siglo XIX cuando se está en el siglo XXI, las aulas de clase todavía están estructuradas en base al método frontal, esto es, una disposición centrada en el frente, con un punto de atención en la figura del docente facilitador de contenidos, y en una tecnología visual como la pizarra, la lámina o el data show, y en otras aulas hay un grupo de escolares que aprenden todos al mismo tiempo las mismas cosas (masificación de la enseñanza), y que atienden a un maestro adulto que plantea un programa unificado y central que organiza al conjunto (como el trívium medieval que consistía en gramática, lógica y retórica para saber que decir y como decirlo), en la que no se hace uso en la mayoría de los casos de las NTICs.

O en el mejor de los casos se hace uso como lo expresa De Miguel (2005)

El profesorado hace uso de la computadora, del proyector para mejorar su explicación y hacer más ágil su clase, se emplean recursos (presentaciones, simulaciones virtuales, contenidos multimedia, videos de youtube, etc), que puede haber preparado el propio profesor o que han sido elaborados por terceros (editoriales u otros profesionales...). (p. 756)

Como se puede apreciar en este último caso el único que se relaciona con el uso de la tecnología es el docente y aunque facilita de alguna manera el aprendizaje del estudiante y lo motiva visualmente pero sigue la misma educación "bancaria" es decir la pedagogía tradicional en que el único protagonista del proceso de enseñanza-aprendizaje es el docente, no se estimula el trabajo cooperativo y dialogante, y además que no fomenta el acceso a las NTICs por parte de los alumnos.

En estas escuelas el enfoque psicologista, que según Bolaños y Molina (2007) "se centra en los análisis psicopedagógicos del individuo” (p. 92), casi no se percibe porque las necesidades, características, intereses, gustos y habilidades de cada estudiante no son casi tomados en cuenta o simplemente son ignorados; en cuanto al enfoque socioreconstruccionista "que se centra en el individuo como realidad socio-cultural y en la sociedad como realidad sistemática e institucional" (p. 92). Ésta se percibe poco en las aulas de clases, a pesar que se invoca sus bondades en las facultades de educación y en las capacitaciones pedagógicas, y esto porque tiene más prestigio en la sociedad peruana el 
enfoque academicista "que se centra en la valoración de contenido cultural sistematizado y en el proceso de transmisión de ese contenido" (p. 92), y esta es la que predomina en la mayoría de las escuelas del Perú y sobre todo en las preuniversitarias, en que el aula y los estudiantes giran alrededor del docente, los contenidos se tornan como un fin en sí mismo y no como un medio para enseñar a pensar, a analizar, a valorar, para crear, para aplicar y para pensar sobre lo que pensamos, este enfoque además deja de lado el conocimiento cotidiano, "que es el que emana espontáneamente de la realidad y de la experiencia que el alumnado vive en forma cotidiana” (Sibilia, 2005, p. 809).

Tomando en consideración lo expresado por Casanova (2012), un grave problema que acarrean nuestro sistema educativo es la gran cantidad de contenidos de carácter conceptual que intentan introducir en las cabezas de los estudiantes. Es decir, la cantidad de información que pretenden que se memoricen sin ni siquiera analizarla, aunque esto no suponga (como ocurre en la mayoría de los casos) ni más aprendizaje real ni mejor preparación para la vida. Sin embargo, las escuelas preuniversitarias hacen esto (aunque desde el año 2014 ya les ha prohibido publicitarse con ese nombre aunque sus prácticas pedagógicas siguen siendo las mismas) y los padres de familia avalan este tipo de enseñanza, porque ellos tienen la percepción que mientras más llenen el cuaderno los profesores de contenido tan buenos serán, aunque esto derive en una educación rutinaria, poco interesante, desmotivadora para sus hijos, y por lo que se deja a muchas personas por el camino de la escuela y de la vida.

En las escuelas peruanas como se pudo apreciar predomina el enfoque academicista que es un currículo excesivamente orientado a los contenidos de la cultura sistematizada y casi poco o en el peor de los casos nada al desarrollo de capacidades socioafectivas o emocionales, y eso a pesar que el grado de aprendizaje depende en gran parte del desarrollo cognitivo, emocional y social del estudiante.

El sistema educativo peruano no hace casi uso de las NTICs, porque en su currículo nacional no se especifica su uso en el salón de clase. Y si se hace uso en algunas escuelas es junto con el enfoque analógico, lineal, dictatorial y memorístico que impera en la actualidad que es el academicista, que da más importancia a los resultados que a los procesos. Lo anterior lo sustenta lo expresado por (OREALC-UNESCO, 2013):

Los modelos educativos y (...) los contenidos que forman parte del currículum actual y (...), en la mayoría de países latinoamericanos, en lo sustancial fueron diseñados para satisfacer las demandas de una sociedad muy distinta, y anterior, a la sociedad del conocimiento. Los cambios vertiginosos de las sociedades contemporáneas ponen en cuestión qué es lo que se debe enseñar y cómo se puede aprender mejor. (p. 9)

Delors (1996) señala que la escuela y los docentes deben asumir un nuevo papel; la escuela debe convertirse en un lugar más atractivo para los alumnos y facilitarles la clave para un mejor entendimiento de la sociedad del conocimiento, al mismo tiempo que los problemas de la sociedad deben entrar en las escuelas y el docente debe hacer entrar en contacto y guiarles en la propuesta y búsqueda de soluciones a estas problemáticas que se presentan en el entorno del estudiante.

La escuela tiene que interactuar con la sociedad y aprender de él, salir de su aislamiento y endogamia, para de esta manera innovar y hacer frente a retos continuos, y en ellos las NTICs tienen un papel estratégico, porque darían una mayor integración de la educación en la dinámica social. 


\section{Calidad y Pertinencia de la Formación Docente y de las Escuelas Peruanas para la Sociedad del Siglo XXI}

Hay una interdependencia muy evidente entre pertinencia y calidad, al punto que se podría decir que una presupone a la otra, como las dos caras de una misma moneda. La pertinencia es una dimensión de la calidad de la educación, aunque pertinencia y calidad deben marchar siempre de la mano, porque los esfuerzos encaminados a mejorar la calidad de la educación no pueden omitir la valoración de su pertinencia, porque ésta se refiere a la adecuación entre lo que la sociedad espera de las instituciones y lo que estas hacen a su servicio (Tünnermann, 2000), pues según la UNESCO (1998) "la pertinencia de la Educación debe evaluarse en función de la adecuación entre lo que la sociedad espera de las instituciones y lo que éstas hacen” (p. 2)

La pertinencia alude a la necesidad de que la educación sea significativa para personas de distintos estratos sociales y culturas, y con diferentes capacidades e intereses, de forma que puedan apropiarse de los contenidos de la cultura, mundial y local, y construirse como sujetos, desarrollando su autonomía y su propia identidad y responder con ellos a las necesidades de su entorno social. Para que haya pertinencia, la educación tiene que ser flexible y adaptarse a las necesidades y características de los estudiantes y de los diversos contextos sociales y culturales.

La Oficina Regional de Educación de la UNESCO para América Latina y el Caribe (OREALC-UNESCO, 2013) propone una primera aproximación de lo que es calidad de la educación. La concibe como "un medio para que el ser humano se desarrolle plenamente como tal, y que le permite crecer y se fortalecerse como persona y (...) que contribuye por ende al desarrollo de la sociedad, transmitiendo y compartiendo sus valores y su cultura" (p. 6).

La educación debe entenderse como un proceso permanente que facilita el aprendizaje, el desarrollo de competencias, la experiencia y la incorporación plena de los valores, afectos y de sus potencialidades, tanto individuales como sociales. Así, tiene un valor en sí misma y no únicamente como herramienta para el crecimiento económico o el desarrollo social. La misión de la educación es el desarrollo integral de ciudadanos que también sean capaces de transformar la sociedad actual, haciéndola más justa, inclusiva y democrática, más que la formación de sujetos capaces de integrarse y "funcionar adecuadamente" en ella (mera función reproductiva de la educación).

En el Perú, la mayoría de las universidades no están formando docentes para la sociedad del siglo XXI, un claro ejemplo de esto es que la Facultad de Educación de la Universidad Nacional José Faustino Sánchez Carrión no ha rediseñado su currículo desde el año 1999, por lo que los futuros docentes procedentes de esta facultad de educación no están siendo preparados para hacer uso de las NTICs en el aula de clases. A pesar de que estas según Palomo y otros (2006) "se han convertido en un instrumento cada vez más indispensable en los centros educativos” (p. 18) pero sobre todo en los procesos de enseñanza aprendizaje, porque son una herramienta estratégica que apunta a mejorar la calidad de la educación, la cual daría la oportunidad de una constante interacción de parte del alumno hacia sus actividades y que investigue los contenidos de sus tareas, sin embargo, estos son ignorados en los planes de estudios de la formación docente. 
Además que la introducción de las NTICs en las aulas pone en evidencia la necesidad de una nueva definición de roles, especialmente, para los alumnos y docentes. Los primeros, porque pueden adquirir mayor autonomía y responsabilidad en el logro de sus aprendizajes, lo que obliga al docente a salir de su rol anacrónico de única fuente de conocimiento, aunque esto le genera incertidumbres, tensiones y temores; sin embargo la realidad del siglo XXI obliga a una readecuación creativa de la institución escolar y del rol docente en el proceso de enseñanza-aprendizaje.

"Los estudiantes de las escuelas en su mayoría son nativos digitales, pues se trata de jóvenes que no han conocido el mundo sin Internet, y para quienes las tecnologías digitales son mediadoras de gran parte de sus experiencias" (OREALC-UNESCO, 2011, p. 9). Aunque la formación de los docentes y las prácticas en las aulas de clases en todos los niveles educativos sigue anclada principalmente en el siglo XIX.

El Ministerio de Educación del Perú ha realizado algunos esfuerzos por incorporar los NTICs, y entre las más importantes tenemos los programas Huascarán (2002), Una Laptop por Niño (2006) y el último la Dirección General de Tecnologías Educativas (DIGETE) (2012).

Sin embargo, la incorporación de estas, sólo ha sido la de "importación", introduciendo en las escuelas: dispositivos, cables y programas computacionales, sin una claridad previa acerca de cuáles son los objetivos pedagógicos que se persiguen, qué estrategias son las apropiadas para alcanzarlos y, que se verían plasmados en el currículo nacional y el resultado de todo lo anterior es que las tecnologías han ocupado un lugar marginal en las prácticas educativas, las que siguen siendo relativamente las mismas que había antes de la implementación de los programas antes mencionados.

Lo anterior lo confirma lo expresado por Sandro Marcone (actual director de la DIGETE y ex director del proyecto Huascarán), para quien según la experiencia que tuvo en los dos programas que dirigió el énfasis de la gestión se ponía inicialmente en la provisión de tecnología educativa y no en conocer y facilitar las dinámicas de apropiación y uso de las mismas. Esto ha llevado a que haya una debilidad importante en cuanto a la evaluación de resultados, pues las evaluaciones se han concentrado más en los objetivos de cobertura (entrega de computadoras, por ejemplo) "que en el impacto de las TICs en los aprendizajes o gestión de las escuelas” (Balarin, 2012, p. 21).

Los docentes que se oponen a cambiar sus metodologías de enseñanza, sin darle espacio al uso y aprovechamiento de las tecnologías, están limitando sus posibilidades de encontrar nuevas rutas para facilitar experiencias educativas distintas e innovadoras, que potencien el aprovechamiento de otros medios para desarrollar el pensamiento crítico en los estudiantes, porque estas les facilitará en gran manera el análisis y el encontrar diferentes alternativas de solución a los problemas de la sociedad, tales como las relaciones humanas, de la programación televisiva, del deporte, de la pobreza, de la violencia de género, de la inseguridad ciudadana, de la vida política y social, y convertirlas en temas de reflexión y de análisis, y eso es lo que la escuela tiene que enseñar fundamentalmente: a analizar los problemas, y que a la vez motivará el trabajo colaborativo y el fortalecimiento de las habilidades comunicativas e informativas.

Las resistencias de la incorporación de nuevas tecnologías en la educación ya se ha visto en el siglo XVI y XVII, lo cual según Drucker (1994) se convirtió en un factor principal en la decadencia de la China y del Islam y su posterior supeditación al occidente, pues 
sólo se centraron en la memorización. En cambio el occidente pasó a una posición de liderazgo mundial, porque en gran parte se reorganizó las escuelas en torno a la nueva tecnología del libro impreso.

Para que se formen docentes con calidad y pertinentes a la sociedad del siglo XXI, las facultades de educación deben formar una nueva generación de profesores de acuerdo a los nuevos paradigmas educativos, que sean competentes en los contenidos de sus asignaturas, en la aplicación de estrategias pedagógicas apropiadas a su asignatura, con conocimientos en psicopedagogía y en el uso eficiente de las NTICs.

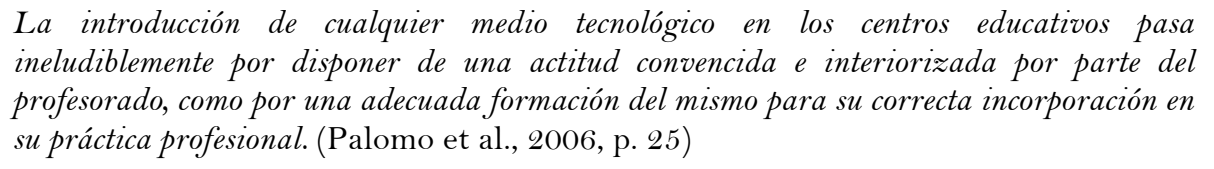

Sobre todo que estos futuros docentes sean flexibles al cambio y con una actitud favorable al aprendizaje permanente que la sociedad actual demanda. Porque las NTICs juegan un papel muy importante en la educación, tal como se aprecia en la ayuda que brindan a los estudiantes para que estos aprendan a su ritmo y en su tiempo; estas sirven de guía a estudiantes y profesores, facilitan la adquisición de todo tipo de recursos de audio, video y datos, y, tal vez lo más importante, permiten la interacción entre ellos mismos.

Uno de los retos principales de los docentes en el siglo XXI radica en comprender al estudiante contemporáneo: creativo, móvil, multi-tarea, colaborativo y productor, que además está acostumbrado a obtener conocimientos procesando información discontinua y no lineal, de ahí la necesidad de dar más énfasis al aprender, más que en la enseñanza como tal, y una clave para lograrlo es el aprendizaje colaborativo mediante el uso de las NTICs.

\section{El Nuevo Rol Docente en la Sociedad del Siglo XXI}

Una dificultad que hay en el aula de clases es que los alumnos no pueden aplicar los conocimientos impartidos a contextos reales, y esto más que nada se debe a que muchos docentes todavía aplican la memorización como parte del aprendizaje de los alumnos sin comprobar si éste ha comprendido, porque repetir de memoria un concepto no demuestra que un estudiante haya entendido. Por lo que el docente debe aplicar estrategias que ayuden al estudiante a analizar y a reflexionar sobre el conocimiento que se va adquiriendo y que permita a la vez modificar y desarrollar su estructura cognitiva.

Los profesores son los que ponen las condiciones para que los estudiantes aprendan mediante su propia actividad; sabemos que el conocimiento tiene que ser construido por el propio sujeto, tiene que asimilarlo y acomodarse a él. Entonces, el profesor lo que tiene que hacer es facilitar el aprendizaje, crear las situaciones en las cuales el alumno aprenda a partir de su propia actividad y experiencia.

El docente ya no sería sólo un proveedor o facilitador de contenidos (porque para desempeñar esa función tenemos a la computadora y a la biblioteca) y en este caso el docente estaría de más en el aula de clase; sino que su función principal en el aula de clase debe ser el promover y desarrollar los procesos de aprendizaje de los alumnos para hacerlos pensar, reflexionar, investigar, comprender y que sean estratégicos, y además debe contextualizar el conocimiento para que el estudiante pueda relacionarlo con su 
entorno inmediato y de esta manera lograr un aprendizaje significativo. Además de hacer útil el conocimiento, porque sólo así se logrará incidir en el desarrollo integral del alumno y también de su entorno social, lo que permitirá alcanzar el propósito esencial de la educación que es incorporar al individuo a su medio social con posibilidades de éxito y además de enriquecerlo y transformarlo.

Entre los recursos que el docente puede utilizar están: los blogs, las redes sociales, los foros, las wikis, las webquest o su variante las miniquest, que están generando espacios virtuales de aprendizaje no formal que el sistema educativo peruano, en general, no aprovecha.

Las estrategias que el docente puede utilizar junto con las herramientas antes mencionadas están: el del Aprendizaje Basado en Problemas (ABP), el uso de la analogía o la V de Gowin, los cuales lograrán que los estudiantes desarrollen las siguientes habilidades: toma de conciencia, pensamiento crítico, pensamiento reflexivo, pensamiento creativo, pensamiento estratégico, desarrollo de habilidades en la lectura de comprensión, trabajo autónomo, trabajo en equipo, responsabilidad en su rol, aprender a pensar, interacción con sus compañeros, adquisición de nuevos conocimientos, aplicación de estrategias de investigación y solución de problemas.

Es necesario que los docentes busquen aplicar estas estrategias junto con las herramientas anteriormente propuestas para que el estudiante se relacione más con entornos reales y se haga menos abstracta la explicación de los conceptos. Es muy importante también que el educador considere las experiencias de aprendizaje previas que el alumno ha vivido, tanto fuera como dentro de la escuela.

El uso de las NTICs en el aula si son utilizados adecuadamente traen muchas ventajas tanto en los alumnos como en los docentes, pero un problema muy común en la educación peruana es que el uso de las NTICs se puede confundir con el uso que hace el docente de una pizarra digital o el uso de otros recursos para la mejor exposición de las clases de los docentes y en la que los alumnos no tienen ningún acercamiento al uso de estos recursos tecnológicos; o que se den clases de informática a los alumnos de forma aislada y totalmente descontextualizado o no relacionados con otros cursos o materias, que según De Miguel (2005) no aportan un sentido funcional a los conocimientos y destrezas que se requieren porque es una actividad que no está estrechamente relacionado con el resto del trabajo escolar por lo que no se daría un aprendizaje significativo a los alumnos ni podrían darle un uso estratégico y reflexivo a estos recursos tecnológicos.

Se pueden confundir también el uso de las NTICs con el uso de softwares educativos en el aula de clases, que aunque su uso puede ser más atractivos que los ejercicios tradicionales de lápiz y papel, sin embargo según De Miguel (2015) "no se estaría estimulando en los alumnos y los docentes el uso de la tecnologías para mejorar su capacidad de buscar, procesar y elaborar información, o para acceder a las nuevas formas de comunicación e interacción” (p. 759). Y el alumno tendría la misma actitud pasiva, pues no estaría en una actividad constante, en una búsqueda y replanteamiento continuo de contenidos y procedimientos (Palomo et al., 2006).

En cambio si se hace un uso adecuado de las NTICs en el aula de clase se estaría proporcionando tanto al docente como al alumno una útil herramienta tecnológica posicionando así a este último en actor protagonista en el proceso de enseñanza 
aprendizaje. De tal forma, según Rodríguez (2009) estaríamos ante una renovación didáctica en las aulas donde se pone en práctica una metodología activa e innovadora que motiva al alumnado en las diferentes disciplinas o materias. Además, que estos recursos aumentan la posibilidad de interactuar facilitando el aprendizaje significativo. Por otra parte Palomo y colaboradores (2006) también considera que estos recursos tienen un alto poder de motivación que predispone a los estudiantes a ser protagonistas de su propio aprendizaje y aumenta su atención e interés por las tareas.

Un uso adecuado de los recursos NTICs en el aula debe desarrollar en los estudiantes competencias en el procesamiento y manejo de la información y que, como lo expresa Palomo y sus colaboradores (2006) "aumenten la implicación del alumnado en sus tareas y desarrollen su iniciativa, y que se vean obligados constantemente a tomar decisiones, a escoger, seleccionar y filtrar información” (p. 18). ¿Pero cómo sabemos que lo que se hace en el aula de clases con los recursos tecnológicos es el adecuado? Según De Miguel (2005) debe haber un uso constante de las computadoras y/o de Internet como fuente de información: buscadores, enciclopedias, y otros materiales de consulta e investigación (como las denominadas webquests u otros similares o páginas web en las que el profesor da algunas pautas para guiar el trabajo de búsqueda del alumnado, incluyendo la consulta, estudio crítico y elaboración de trabajos a partir de recursos variados entre los que se incluyen determinadas direcciones de Internet). Esto permitiría que el alumno puede acceder fácilmente a una retroalimentación de lo estudiado y aprendido en clase, aprender de sus errores, ensayar una y otras vez respuestas alternativas con gran facilidad y podrá emprender nuevos caminos para superar inconvenientes surgidos. Tal como lo expresa Rodríguez (2009) "es mucho más sencillo corregir los errores que se producen en el aprendizaje, puesto que éste se puede corregir en el momento sin necesidad de que el profesor esté pendiente de dicho proceso" (párr. 14).

Según de Miguel (2005) esto además debe promover tanto el posicionamiento crítico como el compromiso con el entorno social, a la vez de ofrecer variadas oportunidades de participación del alumnado en la comunidad y de la comunidad en el centro escolar. Por lo cual el uso de NTICs en el aula de clase no debe ser ajena a lo que suceda en el entorno social del alumno. Además Sibilia (2005) agrega:

\section{El conocimiento debe poder relacionarse y aplicarse en contextos reales y así se logrará un aprendizaje significativo, porque el alumno le daría sentido a la información y se alejaría de esta manera de una aplicación memorística-mecanicista, que conduce a la pérdida del sentido de la aplicación del conocimiento, alejando la escuela de la vida misma. (p. 809)}

En el internet abunda la información, en el que cada día se transfieren el equivalente a 300 millones de páginas, ante eso el docente debe implementar estrategias haciendo uso de las NTICs primero para confrontar esa información y luego que los alumnos intercambien ideas, discutan y decidan en común el porqué de tal opinión, y luego busquen que esa información sea validada relacionándola con situaciones reales y de esta manera el alumno construirá su propio conocimiento con ayuda de sus iguales y con asesoría constante del docente.

De Miguel (2005) también agrega que esta debe tener la elaboración de trabajos y todo tipo de producciones utilizando diversas herramientas informáticas y/o multimedia. Que haya presentaciones del alumnado a su grupo. Que haya un uso constante de medios de comunicación (radio, correo electrónico...) como recurso educativo. En general, todo tipo de trabajo por proyectos de aprendizaje gestionados por el alumnado con el profesorado actuando como guía. 
Todo esto favorecerá el trabajo colaborativo entre los alumnos, el trabajo en grupo, pues los medios informáticos estimularán actitudes como ayudar a los compañeros, intercambiar información relevante encontrada en Internet, resolver problemas a los que los tiene (Palomo et al., 2006). Además que los alumnos serán más autónomos con respecto al docente porque por ellos mismos podrán resolver los problemas que se les presenten en el camino a lograr su aprendizaje.

También habría una serie de ventajas para el profesorado, según Perè Marqués (citado en Palomo et al., 2006) que entre los principales tenemos: representan una fuente de recursos educativos para la docencia, difícilmente sustituibles por otros más tradicionales, permiten al profesorado una individualización de la enseñanza, pues la computadora puede adaptarse a los conocimientos previos del alumnado y a su ritmo de trabajo, les liberan de trabajos repetitivos, monótonos y rutinarios. Por ejemplo, la corrección de operaciones matemáticas, de ejercicios de ortografía se les puede encomendar a la computadora, por lo que pueden dedicarse a la atención personalizada de alumnos que más lo necesiten.

El uso adecuado de las NTICs en el aula de clase trae una serie de ventajas que estarían ayudando a formar al estudiante que es necesario en la sociedad del siglo XXI, pues ante la cantidad la información que se dispone a través de internet, resulta hasta descabellado que el docente solo se dedique a ser transmisor de contenidos y en cambio como expresa Sáez (2012) "(El uso adecuado) de las tecnologías aportan grandes posibilidades de impulsar el Aprendizaje Constructivista y mejorar los procesos de enseñanza aprendizaje" (p. 59). Por lo que el docente mediante el uso de las NTICs debe buscar que los alumnos desarrollen habilidades en la búsqueda y selección de información obtenidas de diversas fuentes para confrontarlos y luego validarlas mediante la relación que se haga con su entorno social, y de esta manera estará dando una formación pertinente y de calidad porque estaría dando respuestas pertinentes a los estudiantes y a su entorno social y así aportará para que la educación transforme las condiciones de toda la sociedad peruana en su conjunto y en todos los ámbitos.

\section{Lo que la Escuela Debe Promover en sus Estudiantes}

El desarrollo social y económico del siglo XXI exige que los sistemas educativos ofrezcan nuevas habilidades y competencias a los estudiantes, y de esta manera beneficiar en primer lugar al educando y luego a la sociedad porque se contribuirá activamente al desarrollo social, cultural y económico bajo un sistema cuya principal base es el conocimiento.

Ante esta dinámica de la sociedad actual, los modelos pedagógicos tradicionales están en crisis. De esquemas lineales, autoritarios, analógicos, se está pasando a modelos en red, participativos y digitales. La unidisciplinariedad es sustituida por la convergencia e interacción entre múltiples disciplinas. La enseñanza y el aprendizaje in situ van sin quedan atrás frente a las alternativas de trabajo virtual y compartido que se pueden dar en el aula de clases.

La necesidad de cambiar los modelos pedagógicos tradicionales para dar respuesta a los nuevos tiempos lo menciona el informe Delors (1998) el cual expresa que el estudiante debe aprender a aprender, aprender a ser, aprender a hacer, aprender a convivir y todo esto relacionado con que el estudiante sea crítico, creativo y colaborativo, lo cual se 
estimularía enormemente con el uso de la NTICs en el aula de clases. Porque éstas según Palomo y colaboradores (2006) “(...) representan un recurso cada día más imprescindible en nuestra vida, unas herramientas adecuadas para la preparación presente y futura del alumnado" (p. 12).

Por lo que la concepción de la educación como simple transmisión acumulación de información debe ser superada en los centros educativos peruanos y para dar respuesta al informe Delors y sobre todo a la sociedad del conocimiento, el aprendizaje debe apuntar a la colaboración, la participación y la creatividad.

Palomo et al. (2006) expresa:

Los centros educativos tienen que preparar a su alumnado para, no sólo acceder a la información, sino también saber "crear" conocimiento basado en dicha información. Deben saber filtrar, seleccionar, valorar, criticar, desechar, utilizar adecuadamente dicha información, a la que tienen acceso. $\Upsilon$ todo este proceso va a llevarles a una preparación imprescindible para su futuro en la sociedad en la que nos encontramos. (p. 12)

Entonces más que el interés en el producto final del conocimiento, el énfasis actual debe estar en los procesos de aprendizaje, es decir en cómo se llega a ese conocimiento y que este sea pertinente.

\section{El Nuevo Modelo Educativo para la Educación Peruana}

En el Perú, se plantea un cambio urgente para estar acorde a los nuevos tiempos y eso depende se considera más que nada de la sociedad, pues ante ella, las escuelas preuniversitarias (en las que predomina el enfoque academicista, porque se valora más la transmisión del contenido de la cultura sistematizada sin un análisis previo de esta por parte del alumno) todavía tienen mucho prestigio, y mientras haya esto, estas escuelas estarán en su zona de confort y no buscarán reorientar su enfoque curricular y pedagógico.

Se requiere urgente una orientación del Modelo Educativo en todo el sistema educativo peruano porque no estaría acorde al siglo XXI, así que en esta se debe considerar el enfoque psicologista, porque las necesidades, los intereses, contexto, características personales y gustos de cada estudiante deben ser tomados en cuenta, construyendo de esta manera, las experiencias significativas de su propio aprendizaje, lo que en las escuelas del sistema educativo peruano, en la mayoría de los casos no se hace porque lo único que buscan es la trasmisión de contenidos en masa y formar personas sin criterio propio o capacidad de crítica (tal como se hacía en la sociedad industrial); también se debe considerar en el nuevo modelo educativo el enfoque socio-reconstruccionista, que pretende transformar la educación en un proceso de socialización o culturalización de la persona, y que el contenido no sea un fin en sí mismo sino solo un medio para lograr otras competencias necesarias para las personas del siglo XXI y por último se debe considerar el enfoque tecnológico el cual mejorará ostensiblemente ese proceso de socialización y culturalización y los procesos de enseñanza-aprendizaje en la medida que creará el escenario para la creatividad, la colaboración y la innovación; y permitirá hallar nuevas y mejores opciones para atender diferentes necesidades educativas de los estudiantes y ampliará las posibilidades de formar ciudadanos críticos. 
En este nuevo modelo educativo el estudiante debe aprender a comprender y no sólo a memorizar, pues le debe dar significado a lo que aprende y su posterior aplicación. El docente debe dar significatividad a los datos e informaciones que ofrece al estudiante, y más que conocimientos (sin dejarlos de lado) debe estar más preparado en psicopedagogía, para identificar fácilmente los estilos de aprendizaje de los estudiantes, de cómo aprenden mejor y como estos construyen su aprendizaje, y en el uso eficiente de las NTICs.

Porque las NTICs favorecen el trabajo colaborativo con los iguales, pues estimulan actitudes como ayudar a los compañeros, intercambiar información relevante encontrada en Internet, a resolver problemas a los que los tienen. Estimula a los integrantes de los grupos en el aula de clase a intercambiar ideas, a discutir y decidir en consenso sobre la respuesta adecuada a un asunto.

\section{Conclusiones}

Las escuelas peruanas están formando a los estudiantes con las características y demandas del siglo XIX, es decir, para una sociedad industrial, y no con los de la sociedad del siglo XXI, siendo de esta manera una educación de poca calidad y pertinencia.

La implementación y uso en el ámbito educativo de las nuevas tecnologías de la información y comunicación (NTICs), se han vuelto una necesidad sine qua non, pues tienen un papel crucial en el logro de las competencias necesarias en los estudiantes del siglo XXI. Sin embargo, la inadecuada, poca o nula implementación de los NTICs lleva a que en la educación peruana predomine aún el enfoque academicista, que consiste esencialmente en la mera reproducción de los conocimientos de la cultura sistematizada en los estudiantes sin que estos realicen un análisis previo de los contenidos, además que los estudiantes están inmersos en un proceso de enseñanza-aprendizaje centrada en el docente sin que puedan ser los protagonistas de su propio aprendizaje y en la forma de usar, construir y divulgar el conocimiento.

El enfoque academicista predomina en las escuelas, a pesar de los diversos lineamientos y orientaciones del Ministerio de Educación, y además que este enfoque tiene más prestigio en la sociedad peruana pues los padres de familia piden resultados concretos y rápidos, y que puedan entender y validar, como por ejemplo que el niño pueda leer de corrido en inicial, escribir correctamente en primer grado o que la escuela secundaria brinde cursos preuniversitarios en la secundaria. Por esto es que las escuelas peruanas no contribuyen al desarrollo integral de los estudiantes y se han convertido, en cambio, en un obstáculo para ello, tal como lo hacen hoy en día, estas escuelas autollamadas preuniversitarias, que más que nada buscan un objetivo cortoplacista que es el ingreso del alumno a la universidad y se descuida otras áreas cruciales de la formación del estudiante que le daría las capacidades suficientes para poder enfrentar con éxito el estudio de su carrera y posterior desempeño profesional.

Y por último los diseños curriculares de las Facultades de Educación están desfasadas y muchas de ellas tienen más de 15 años de haberse implementado por lo que los futuros docentes no tendrían las competencias necesarias para hacer que la escuela responda efectivamente a las características y demandas de la sociedad del siglo XXI y específicamente de los estudiantes, siendo por lo tanto una formación no pertinente. 


\section{Sugerencias}

El sistema educativo peruano debe propiciar que los estudiantes tengan la capacidad de investigar, la capacidad de aprender y desaprender permanentemente y de lograr habilidades para buscar, procesar y analizar la información procedente de diversas fuentes, para así llegar al conocimiento, y así responder de manera adecuada a los demandas tan cambiantes de la sociedad del siglo XXI.

El Ministerio de Educación conjuntamente con otros entes gubernamentales y no gubernamentales debe plasmar en el currículo nacional el uso de las nuevas tecnologías de la información y comunicación (NTICs) en el proceso de enseñanza aprendizaje, porque el uso de estas tecnologías estarían dejando de lado la memorización de contenidos o lo que Freire (2008) llama la educación bancaria y en cambio mediante el uso adecuado de estas tecnologías estaríamos posibilitando un aprendizaje significativo en los alumnos y que contribuya a que comprendan lo que están estudiando y lo relacionen con sus experiencias previas, su entorno social y con su visión del mundo.

Las facultades de educación deben rediseñar sus currículos y planes de estudio para de esta manera formar docentes que puedan responder a las demandas y características del siglo XXI y la de los mismos estudiantes y así puedan formar a estos últimos con calidad y pertinencia; además las facultades de educación conjuntamente con el Ministerio de Educación, deben buscar que las escuelas reflejen en la práctica docente lo que se expresa en su currículo institucionalizado y en el currículo nacional. Y que en el aula de clase se debe dar la llamada revolución copernicana, es decir, el centro del proceso de enseñanza aprendizaje deben ser los estudiantes y su aprendizaje y no el docente y su enseñanza, convirtiéndose éste último en un facilitador pero no de contenidos sino de aprendizajes.

\section{Referencias}

Balarin, M. (2012). Programa TIC y educación básica, políticas TIC en los sistemas educativos de América Latina: Caso Perú. Buenos Aires: UNICEF.

Bolaños, G. y Molina, Z. (2007). Introducción al currículo. San José: UNED.

Casanova, M. (2012). El diseño curricular como factor de calidad educativa. REICE. Revista Iberoamericana sobre Calidad, Eficacia y Cambio en Educación, 10(4), 6-20.

Delors, J. (1996) La educación encierra un tesoro. Recuperado de: http://www.unesco.org/

De Miguel, C. (2005). Criterios de innovación para la integración curricular de las tecnologías de la información y la comunicación en el aula. REICE. Revista Iberoamericana sobre Calidad, Eficacia y Cambio en Educación, 3(1), 750-763.

Drucker, P. (1994). La sociedad postcapitalista. Bogotá: Editorial Norma.

Freire, P. (2008) Pedagogía del oprimido. Madrid: S.XXI Editores.

Gibbons M., Limoges, C., Nowothy, H., Schwartzman, S., Scott, P. y Trow, M. (1998). La nueva producción del conocimiento (la dinámica de la ciencia y la investigación en las sociedades contemporáneas). Madrid: Ediciones Pomares-Corredor S.A.

González, J. y Santamaría, R. (2013). Calidad y acreditación en la educación superior: integración e internacionalización de América Latina y el Caribe. Revista Educación, 22(43), 131-147

OECD (2003). Education and the economy in a changing society. París: OECD. 
Palomo, R., Ruiz, J. y Sánchez, J. (2006). Las TIC como agentes de innovación educativa. Granada: Junta de Andalucía.

Rodríguez, E. (2009). Ventajas e inconvenientes de las TICs en el aula. Cuadernos de Educación y Desarrollo, 1(9), 45-61.

Sáez, J. (2012). La práctica pedagógica de las tecnologías de la información y la comunicación y su relación con los enfoques constructivistas. REICE. Revista Iberoamericana sobre Calidad, Eficacia y Cambio en Educación, 10(1), 58-73.

Sibilia, M. (2005). Tecnología educativa constructivista-humanista. REICE. Revista Iberoamericana sobre Calidad, Eficacia y Cambio en Educación, 3(1), 799-816.

Tünnermann, C. (2000). Pertinencia social y principios básicos para orientar el diseño de políticas de educación superior. Educación Superior y Sociedad, 11(1), 181-196.

Tünnermann, C. (2011). La universidad del futuro. Managua: Hispamer.

Tünnermann, C. (2012). La universidad búsqueda permanente. Managua: Hispamer.

UNESCO (1998). Conferencia mundial de educación superior en el siglo XXI. Recuperado de: http://unesdoc.unesco.org/images/O011/001163/116345s.pdf

UNESCO-OREALC (2011). Educación de calidad en la era digital. Una oportunidad de cooperación para UNESCO en América Latina y el Caribe. Buenos Aires: UNESCO

UNESCO-OREALC (2013). Enfoques estratégicos sobre las TICs en educación en América Latina y el Caribe. Santiago: UNESCO. 